Internist 2008 • 49:349-352

DOI 10.1007/s00108-007-2007-6

Online publiziert: 23. Januar 2008

(c) Springer Medizin Verlag 2008

\section{Rubrikherausgeber}

K. Werdan, Halle/Saale

\author{
E. Burri ${ }^{1}$ M. Decker ${ }^{2}$ U. Eriksson ${ }^{1} \cdot$ P. Buser ${ }^{3} \cdot$ L. Hunziker ${ }^{1}$ \\ ${ }^{1}$ Innere Medizin, Universitätsspital Basel \\ ${ }^{2}$ Hämatologie, Universitätsspital Basel \\ ${ }^{3}$ Kardiologie, Universitätsspital Basel
}

\title{
28-jähriger Patient mit erfolgreich behandelter dilatativer Kardiomyopathie
}

Die persönliche Anamnese ergab, dass er seit einem Jahr wegen eines hypogonadotropen Hypogonadismus mit Testosteroninjektionen behandelt wurde. Der Patient war vor vielen Jahren aus dem Kosovo immigriert und Vater zweier gesunder Töchter von 5 und 8 Jahren.

\section{Körperliche Untersuchung}

Der Patient war in reduziertem Allgemeinzustand. Der Blutdruck lag bei 105/60 mmHg, die Herzfrequenz bei 115/ min, es bestand eine absolute Arrhythmie sowie eine Orthopnoe. Die Halsvenen waren gestaut, es fanden sich feuchte Rasselgeräusche über beiden Lungenfeldern und Unterschenkelödeme beidseits. Kardial war ein 3/6-Holosystolikum in Mitralposition auskultierbar. Der restliche Status war unauffällig.

\section{Klinische Chemie und technische Untersuchungen}

Das Troponin T war mit o,03 ng/ml (normal $<0,1)$ bei Eintritt und im Verlauf normal. Das BNP war mit $718 \mathrm{pg} / \mathrm{ml}(<50)$ deutlich, die Leberparameter mit ASAT $81 \mathrm{U} / \mathrm{l}(10-50)$ und ALAT $139 \mathrm{U} / \mathrm{l}(10-37)$ leicht erhöht und das TSH mit 1,43 mIU/ $1(0,332-4,449)$ normal. Das Blutbild war unauffällig und das CRP mit $23 \mathrm{mg} / \mathrm{l}(<5)$ erhöht.

Im EKG zeigte sich ein tachykardes Vorhofflimmern, im konventionellen Thoraxröntgenbild fand sich eine Kardiomegalie mit bilateralem Lungenödem und häufiger Erkrankungen.

Lassen Sie Ihre Kolleginnen und Kollegen an Ihren Erfahrungen teilhaben und reichen Sie Ihre „Alltagsfälle", an die sich das evidenzbasierte diagnostische und therapeutische Vorgehen anschließen sollte, bei DER INTERNIST zur Publikation ein!

Wir freuen uns auf Ihre Fälle!

Bitte schicken Sie Ihre Kasuistiken 2 fach ausgedruckt und auf $C D$ direkt an den Rubrikherausgeber

Prof. Dr. K. Werdan

Klinikum Kröllwitz

Innere Medizin III

Ernst-Grube-Str. 40

06097 Halle/Saale

karl.werdan@medizin.uni-halle.de

Die Autorenrichtlinien finden Sie unter www.DerInternist.de

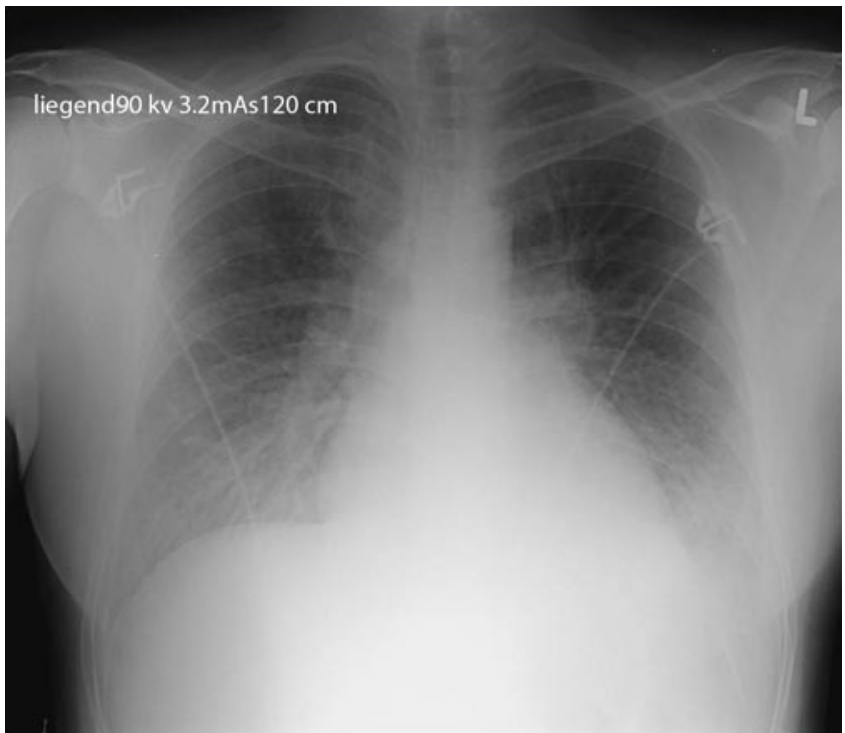

Abb. 1 Konventionelles Thoraxröntgenbild: Kardiomegalie (Herz-Thorax-Quotient 0,55 ), bilaterales alveoläres Lungenödem, Pleuraerguss beidseits 

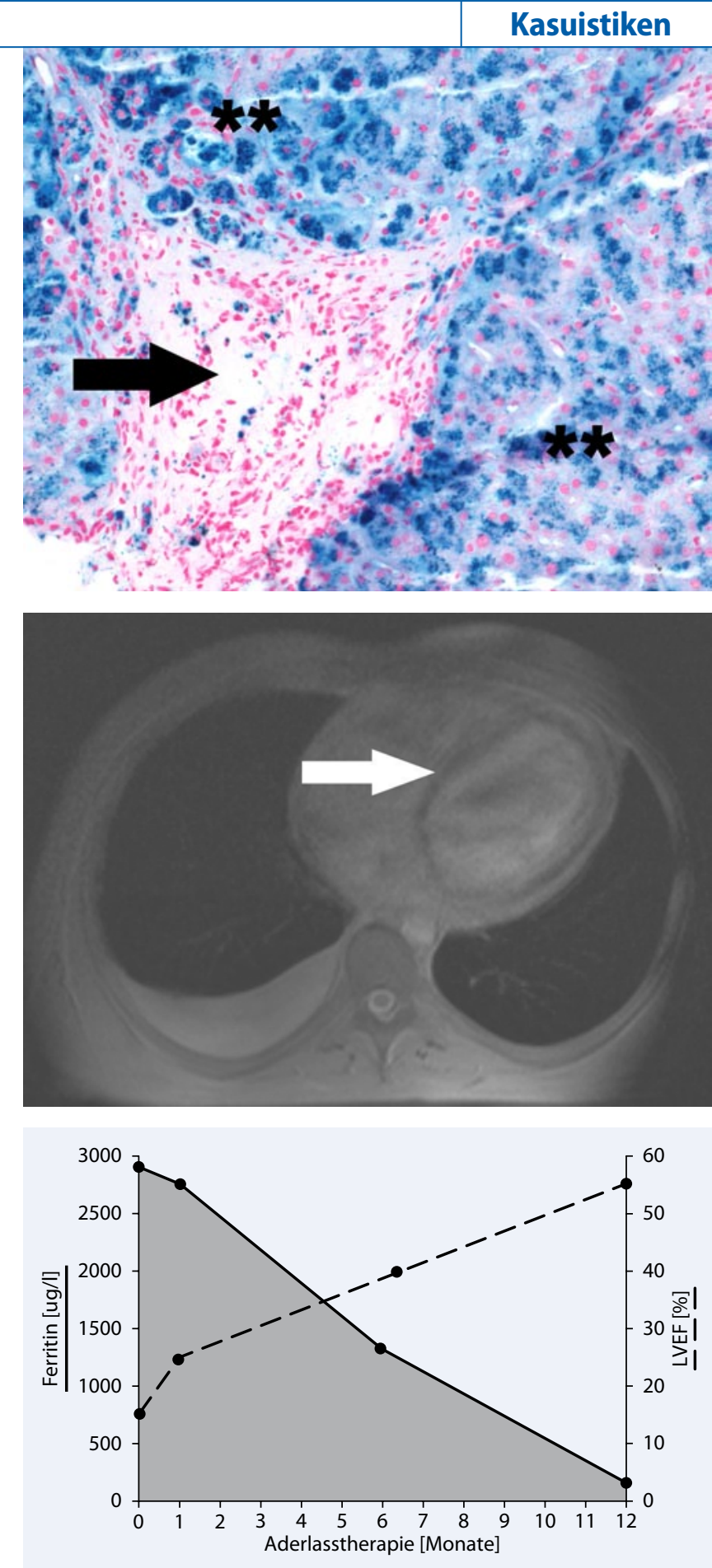

Abb. $2<$ Leberbiopsie (Berlinerblau, 1:20): schwere Hämosiderinablagerungen Grad IV $\left.{ }^{* *}\right)$ in Leberzellen, Sternzellen, portalen Makrophagen und Gallengangsepithel, schwere Portalfibrose mit portoportalen Septen (Pfeil)

Abb. $3<$ Magnetresonanztomographie des Herzen (axiale T1wSpinechosequenz): Dilatation des linken Ventrikels mit ausgedünntem Myokard, homogene Hypointensität des Myokards als Zeichen der Eiseneinlagerung (Pfeil)

Abb. $4<$ Kontinuierliche Verbesserung der linksventrikulären Ejektionsfraktion (LVEF) von $<20 \%$ auf $55 \%$ unter Aderlasstherapie innerhalb von 12 Monaten. Reduktion von Ferritin im gleichen Zeitraum von $2900 \mu \mathrm{g} /$ I auf $149 \mu \mathrm{g} / \mathrm{l}$

Pleuraergüssen beidseits ( $\bullet$ Abb. 1). Die transthorakale Echokardiographie dokumentierte eine Dilatation aller Herzabschnitte mit mittelschwerer Mitralinsuffizienz. Die linksventrikuläre Auswurffraktion (LVEF) war mit 20\% (normal >55\%) bei diffuser Hypokinesie schwer eingeschränkt.
In den Blutuntersuchungen fiel ein stark erhöhtes Ferritin von 2900 Mg/l (15320) und eine Transferrinsättigung von 96\% (16-45) auf. In der Zusammenschau mit der Vorgeschichte eines sekundären Hypogonadismus wurde eine hereditäre Hämochromatose vermutet und eine Leberbiopsie durchgeführt. Dort fand sich eine ausgeprägte Leberzellsiderose (Grad IV), eine schwere Portalfibrose mit portoportalen Septen sowie ein herdförmig vollständiger nodulärer Umbau (- Abb. 2). Das Eisentrockengewicht betrug 13.286 $\mu \mathrm{g} / \mathrm{g}(500-900)$ und war somit massiv erhöht.

\section{Erweitere Diagnostik}

\section{Kardiale Magnetresonanztomographie}

In der Magnetresonanztomographie stellten sich das Myokard und die Leber stark hypodens dar (• Abb. 3). Die Konstellation ist typisch für eine intrazelluläre Eisenüberladung und bestätigte die kardiale Beteiligung bei der vorliegenden Hämochromatose.

\section{Genetische Abklärung}

Die genetischen Abklärungen für Mutationen im klassischen Hämochromatose- (HFE-) Gen (C282Y, H63D und S65C) waren allesamt negativ. Erst die erweiterte Suche nach Mutationen der juvenilen Verlaufsform der hereditären Hämochromatose fand eine homozygote Mutation G320V im Hemojuvelin- (HJV-) Gen.

\section{Diagnose}

\section{Dilatative Kardiomyopathie bei juveniler Hämochromatose.}

\section{Therapie und Verlauf}

Es wurde mit einer intensiven Aderlasstherapie begonnen. Darunter war nach 6 Monaten das Ferritin bereits auf die Hälfte und nach 12 Monaten auf $149 \mu \mathrm{g} /$ 1 abgefallen. Gleichzeitig hatte sich die LVEF von anfänglich $<20 \%$ nach 6 Monaten auf $39 \%$ verbessert und nach 12 Monaten mit 55\% normalisiert (• Abb. 4). Die Dilatation der Herzhöhlen und die Hypokinesie des Myokards waren rückläufig. Der Patient beklagte einzig noch leich- 
Tab. 1 Hereditäre Hämochromatose

\begin{tabular}{|c|c|c|c|c|c|}
\hline $\begin{array}{l}\text { Gendefekt } \\
\text { (Name/Lokalisation) }\end{array}$ & Vererbung & Häufigkeit & Manifestation & $\begin{array}{l}\text { Organ- } \\
\text { schaden }\end{array}$ & $\begin{array}{l}\text { Schwere- } \\
\text { grad }\end{array}$ \\
\hline $\begin{array}{l}\text { Hämochromatose- } \\
\text { Gen (HFE/6p21.3) }\end{array}$ & \multirow[t]{4}{*}{$\begin{array}{l}\text { Autosomal } \\
\text { rezessiv }\end{array}$} & Häufig & \multirow[t]{2}{*}{$\begin{array}{l}\text { 4.-5. Lebens- } \\
\text { dekade }\end{array}$} & \multirow[t]{2}{*}{ Leber } & \multirow[t]{2}{*}{ Mild } \\
\hline $\begin{array}{l}\text { Transferrinrezeptor } 2 \\
\text { (TfR2/7q22) }\end{array}$ & & Selten & & & \\
\hline Hepcidin (HAMP/19q13.1) & & & \multirow{2}{*}{$\begin{array}{l}\text { 2.-3. Lebens- } \\
\text { dekade }\end{array}$} & \multirow{2}{*}{$\begin{array}{l}\text { Endokrine } \\
\text { Organe } \\
\text { Herz }\end{array}$} & \multirow[t]{2}{*}{ Schwer } \\
\hline Hemojuvelin (HJV/1p21) & & & & & \\
\hline
\end{tabular}

te Atemnot bei sportlicher Aktivität, war ansonsten aber beschwerdefrei.

\section{Diskussion}

\section{Pathogenese}

Die hereditäre Hämochromatose ist eine autosomal rezessiv vererbte Erkrankung, die in der überwiegenden Mehrzahl durch eine klassische Mutation im HFE-Gen hervorgerufen wird. In den letzten Jahren hat sich nun aber gezeigt, dass auch andere, nicht HFE-assoziierte Gendefekte zum Phänotyp der hereditären Hämochromatose führen ( $\bullet$ Tab. 1). Mit dieser Erkenntnis hat sich auch das Verständnis des Eisenstoffwechsels grundlegend verändert.

Heute wird davon ausgegangen, dass ein Zusammenspiel von Hepcidin, einem zirkulatorischen Peptid aus der Leber, mit dem membrangebundenen Eisentransportprotein Ferroportin die zentrale Steuerung im Eisenstoffwechsel bildet. Hepcidin führt zur Internalisierung und Degradation des Ferroportins und damit einem geringeren Eiseneinstrom in den Körper. Bei allen Formen der hereditären Hämochromatose kommt es zu einem Abfall des Hepcidins und somit zu einer unregulierten Eisenaufnahme in den Körper.

Die juvenile Hämochromatose ist eine seltene Variante der hereditären Hämochromatose. Genetisch ist sie eine heterogene Erkrankung mit 2 bekannten Gendefekten auf Chromosom 1 (HJV-Gen, Genprodukt Hemojuvelin) und Chromosom 19 (HAMP-Gen, Genprodukt Hepcidin). Vom HJV-Gen sind mehrere Mutationen bekannt, die weitaus häufigste ist G320V, wie bei unserem Patienten. Hemojuvelin scheint ein Regulator der Hepcidintranskription zu sein [5]. Sehr selten tritt auch eine Mutation im HAMP-Gen auf, was zu einem direkten, vollständigen Ausfall von Hepcidin und somit zu einer sehr schweren Verlaufsform der juvenilen Hämochromatose führt.

Die juvenile Hämochromatose ist charakterisiert durch ein Auftreten von Organschäden bereits in der 2. bis 3. Lebensdekade. Häufig manifestiert sich dabei eine Infertilität aufgrund eines sekundären Hypogonadismus (Eiseneinlagerung in die Hypophyse) als erstes Symptom. Unbehandelt tritt eine dilatative Kardiomyopathie auf, und die Patienten versterben an den Folgen eines Herzversagens oder einer Herzrhythmusstörung.

Das frühe Auftreten endokriner und kardialer Organschäden ist typisch für die juvenile Hämochromatose. Im Gegensatz dazu steht bei der klassischen HFE-assoziierten Variante die Leberzirrhose und nicht die Kardiomyopathie als limitierende Erkrankung im Vordergrund. Eine kardiale Beteiligung kann vorkommen, ist aber selten klinisch relevant, weil die Erkrankung aufgrund des Leberschadens meist vor dem Auftreten kardialer Symptome erkannt und behandelt wird.

\section{Abklärungen}

In den Laboruntersuchungen kann zuerst eine erhöhte Transferrinsättigung und im weiteren Verlauf ein erhöhtes Serumferritin nachgewiesen werden. Eine Leberbiopsie sollte zumindest in der initialen Abklärung durchgeführt werden, um das Ausmaß der Leberschädigung beurteilen zu können. Für die Bestimmung des Eisengehalts in der Leber ist eine Leberbiopsie allerdings nicht notwendig. Der Eisengehalt in der Leber kann heutzutage mit speziellen MRT-Sequenzen zuverlässig er-

\section{Klinische Präsentation}

Internist 2008 - 49:349-352

DOI 10.1007/s00108-007-2007-6

(c) Springer Medizin Verlag 2008

E. Burri · M. Decker · U. Eriksson · P. Buser · L. Hunziker

\section{8-jähriger Patient mit erfolgreich behandelter dilatativer Kardiomyopathie}

\section{Zusammenfassung}

Ein 28-jähriger Patient wurde mit dekompensierter Herzinsuffizienz notfallmäßig zugewiesen. In der Vorgeschichte bestand ein hypogonadotroper Hypogonadismus. Echokardiographisch fand sich eine dilatative Kardiomyopathie. Ein erhöhtes Serumferritin und eine Eisenüberladung in der Leberbiopsie ließen eine hereditäre Hämochromatose vermuten. Die kardiale Beteiligung konnte mittels Magnetresonanztomographie nachgewiesen werden. Genetische Abklärungen ergaben eine homozygote Mutation für G320V und bewiesen eine juvenile Hämochromatose. Eine Aderlasstherapie führte innerhalb eines Jahres zur Normalisierung des Eisenstatus und der kardialen Pumpfunktion.

\section{Schlüsselwörter}

Kardiomyopathie · Eisenüberladung · Hämochromatose $\cdot$ Aderlass

\section{8-year old patient with successfully treated dilatative cardiomyopathy}

\section{Abstract}

A 28-year was admitted with heart failure. His medical history included treatment for hypogonadotropic hypogonadism. Echocardiography showed dilatation of all chambers. Elevated serum ferritin levels and liver biopsy indicated hereditary hemochromatosis. Cardiac iron overload was seen on magnetic resonance imaging. Genetic testing revealed homozygosis for G320 V mutation, confirming the diagnosis of juvenile hemochromatosis. Phlebotomy on a biweekly regimen was started and after twelve months of therapy the patient had normal ferritin values as well as normal ejection fraction on echocardiography.

Keywords

Cardiomyopathy · Iron overload · Hemochromatosis · Phlebotomy 


\section{Hier steht eine Anzeige.}

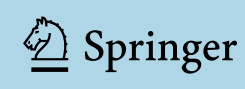

mittelt werden [3]. Auch eine Eiseneinlagerung ins Myokard kann mit der MRT nachgewiesen werden und eine Myokardbiopsie ersetzen [6]. Allerdings ist im Gegensatz zur Leber eine genaue Quantifizierung des Eisengehalts noch nicht möglich.

\section{Therapie}

Therapie der Wahl bei der hereditären Hämochromatose ist die Aderlasstherapie. Der Nutzen einer medikamentösen Eisenchelation, insbesondere bei der juvenilen Verlaufsform, ist unklar und wird nicht empfohlen [1]. Die Erfahrungen basieren fast ausschließlich auf Untersuchungen an Patienten mit Thalassaemia major. Dort konnte gezeigt werden, dass im Gegensatz zu einer Standardeisenchelation mit Deferoxamin, eine Kombinationsbehandlung mit Deferipron den Eisengehalt des Myokards rascher reduzierte und wirkungsvoller die Pumpfunktion des Herzens verbesserte [8]. Auch die Mortalität durch kardiale Ereignisse war unter einer Eisenchelation mit Deferipron geringer als mit Deferoxamin, wohl auch über den Effekt einer besseren Eisendepletion aus dem Myokard [2]. Der Einsatz von Eisenchelatoren ist denjenigen Patienten vorbehalten, bei denen aus hämodynamischen Gründen keine Aderlasstherapie durchgeführt werden kann $[4,7]$.

Die meisten Patienten mit juveniler Hämochromatose werden erst spät, bei Auftreten einer dilatativen Kardiomyopathie, vorstellig. Es ist deshalb entscheidend, das Krankheitsbild zu erkennen und eine Aderlasstherapie zu beginnen. Die Eisendepletion ist für das Überleben entscheidend und muss - wann immer möglich - maximiert werden. Wie unser Fall zeigt, ist auch nach Auftreten einer schweren Herzinsuffizienz eine vollständige Erholung der kardialen Pumpfunktion möglich.

\section{Fazit für die Praxis}

Eisenspeicherkrankheiten und somit auch die hereditäre Hämochromatose gehören in die Differenzialdiagnose von Patienten mit einer dilatativen Kardiomyopathie. Vor allem bei jungen $\mathrm{Pa}$ tienten in der 2. und 3. Lebensdekade muss an das Vorliegen einer juvenilen Hämochromatose gedacht und mittels genetischer Analysen gesucht werden.

\section{Korrespondenzadresse}

Dr. E. Burri

Innere Medizin, Universitätsspital Basel

Petersgraben 4, 4031 Basel

Schweiz

BurriE@uhbs.ch

Interessenkonflikt. Der korrespondierende Autor gibt an, dass kein Interessenkonflikt besteht.

\section{Literatur}

1. Barton JC, McDonnell SM, Adams PC et al. (1998) Management of hemochromatosis. Hemochromatosis Management Working Group. Ann Intern Med 129: 932-939

2. Borgna-Pignatti C, Cappellini MD, De Stefano P et al. (2006) Cardiac morbidity and mortality in deferoxamine- or deferiprone-treated patients with thalassemia major. Blood 107: 3733-3737

3. Christoforidis A, Haritandi A, Tsatra I et al. (2007) Four-year evaluation of myocardial and liver iron assessed prospectively with serial MRI scans in young patients with beta-thalassaemia major: comparison between different chelation regimens. Eur J Haematol 78: 52-57

4. Fabio G, Minonzio F, Delbini P et al. (2007) Reversal of cardiac complications by deferiprone and deferoxamine combination therapy in a patient affected by severe type of juvenile hemochromatosis $(\mathrm{JH})$. Blood 109: 362-364

5. Lin L, Goldberg YP, Ganz T (2005) Competitive regulation of hepcidin mRNA by soluble and cell-associated hemojuvelin. Blood 106: 2884-2889

6. Mavrogeni SI, Markussis V, Kaklamanis L et al. (2005) A comparison of magnetic resonance imaging and cardiac biopsy in the evaluation of heart iron overload in patients with beta-thalassemia major. Eur J Haematol 75: 241-247

7. Nielsen P, Fischer R, Buggisch P et al. (2003) Effective treatment of hereditary haemochromatosis with desferrioxamine in selected cases. $\mathrm{Br} \mathrm{J}$ Haematol 123: 952-953

8. Tanner MA, Galanello R, Dessi C et al. (2007) A randomized, placebo-controlled, double-blind trial of the effect of combined therapy with deferoxamine and deferiprone on myocardial iron in thalassemia major using cardiovascular magnetic resonance. Circulation 115: 1876-1884 